\title{
Validation of Microbiological Testing of Tissue Preparations with Different Incubation Temperatures
}

\author{
Frithjof Herrlinger ${ }^{a}$ Tino Schulz ${ }^{a}$ Axel Pruß $\beta^{a}$ Eva Schulz \\ aUniversity Tissue Bank, Institute for Transfusion Medicine, Charité - Universitätsmedizin Berlin, Berlin, Germany
}

\section{Keywords}

Tissue preparations - Cornea · Femoral head .

Microbiological testing · Temperature $\cdot$ BACTEC $^{\text {TM }}$ FX

\begin{abstract}
Introduction: The European Pharmacopoeia (Ph. Eur.) provides principles for microbiological testing of tissue preparations. According to the Ph. Eur., tests should be performed at different temperatures for detection of aerobic bacteria and fungi $\left(20-25^{\circ} \mathrm{C}\right)$ vs. anaerobic bacteria $\left(30-35^{\circ} \mathrm{C}\right)$. Semiautomated systems using blood culture bottles are already widely used and they are adequate for growth detection. Resin-containing bottles and the addition of penicillinase permit testing of culture media containing antibiotics. $\mathbf{M a}$ terials and Methods: At 3 temperatures $\left(21,30\right.$, and $35^{\circ} \mathrm{C}$ ) cornea culture media with and without dextran (CM II and CM I) and thermal disinfected femoral head medium (FH) were spiked with the 6 reference strains recommended by the Ph. Eur. (additionally: Enterococcus faecalis, Staphylococcus epidermidis, and Cutibacterium acnes). Microbial growth was monitored with the BACTEC ${ }^{\text {TM }}$ FX unit or visually at $21^{\circ} \mathrm{C}$. Results: Growth for all strains was detected with each medium at all 3 temperatures, except for $\mathrm{C}$. acnes at $21^{\circ} \mathrm{C}$ (all media) and $30^{\circ} \mathrm{C}$ with FH. C. acnes had the highest times to detection, requiring test durations of 14 days. Microbial growth was faster at 30 and $35^{\circ} \mathrm{C}$ compared to $21^{\circ} \mathrm{C}$. Conclusion: The requirements according to the $\mathrm{Ph}$. Eur. for a successful method suitability test could be fulfilled for the semiautomated blood culture bottle system with the BACTECTM FX unit for the media and microorganisms used.
\end{abstract}

In the presented validation study $35^{\circ} \mathrm{C}$ was shown to be the incubation temperature with the fastest growth, of the majority of the test strains used, and complete detection within 14 days.

(c) 2020 S. Karger AG, Basel

\section{Introduction}

Human tissue preparations are often used for replacement of tissue defects and need to fulfill high quality standards to be released for transplantation [1]. Microbiological contamination of the transplanted tissue is a potential risk factor for serious complications for the recipient [2] and may, as a worst case scenario, end in the recipient's death $[3,4]$. Since many tissue preparations contain vital cells they cannot be subjected to terminal sterilization treatment by physical or chemical methods without impairing the unique properties.

To minimize the risk of microbiological dangers, many regulations are in place for the procurement of tissue from living and postmortem donors. Rules for donor acceptance are specified, blood testing for infectious diseases is obligatory, and tissue harvesting and processing methods should be done aseptically. Some tissues are exposed to antibiotics in a decontamination process in the tissue bank, which poses the threat of microorganisms surviving this process but not being detected by postdecontamination microbiological testing.

In recent decades, microbiological testing of tissue preparations has become obligatory. The use of blood 
Table 1. Test strains, growth conditions for initial cultivation of the lyophilisates, and CFU of the diluted straines used for inoculation of the blood culture bottles

\begin{tabular}{|c|c|c|c|}
\hline Strain & ATCC No. & Growth conditions & $\mathrm{CFU} / 100 \mu \mathrm{L}$ \\
\hline \multicolumn{4}{|l|}{ Aerobic bacteria } \\
\hline S. aureus ${ }^{1}$ & ATCC 25923 & CBA, overnight & 45 \\
\hline P. aeruginosa ${ }^{1}$ & ATCC 9027 & CBA, overnight & 41 \\
\hline B. subtilis ${ }^{1}$ & ATCC 6633 & CBA, overnight & 32 \\
\hline S. epidermidis & ATCC 12228 & CBA, overnight & 21 \\
\hline E. faecalis & ATCC 51299 & CBA, overnight & 47 \\
\hline \multicolumn{4}{|l|}{ Anaerobic bacteria } \\
\hline C. sporogenes ${ }^{1}$ & ATCC 19404 & CBA, 3 days & 26 \\
\hline C. acnes & ATCC 6919 & CBA, 5 days & 54 \\
\hline \multicolumn{4}{|l|}{ Fungi } \\
\hline C. albicans $^{1}$ & ATCC 10231 & SDA, overnight & 34 \\
\hline A. brasiliensis ${ }^{1}$ & ATCC 16404 & SDA, 9 days & 10 \\
\hline
\end{tabular}

${ }^{1}$ Recommended for validation by the Ph. Eur. CFU, colony-forming units.

culture bottles has been established and advanced as a standard procedure not only in the clinical routine but also during tissue processing [5-8]. In Germany, pharmaceutical laws mandate quality controls for all tissue preparations, and in Europe specifically microbiological testing should be done according to the European Pharmacopoeia (Ph. Eur.) and the guidance of the European Directorate for the Quality of Medicines (EDQM). The test methods used must be validated and adequate for the precise procedures used on the tissue. They are described in Ph. Eur. chapter 2.6.27. In order to test for relevant anaerobic and aerobic bacteria as well as fungi, the use of different incubation temperatures for maximum of sensitivity is recommended $[9,10]$. Modern semiautomated systems work in temperature ranges above $30^{\circ} \mathrm{C}$; therefore a second system is needed to conduct testing at a lower temperature. This study investigated validation of microbiological testing of tissue preparations according to the Ph. Eur. at different incubation temperatures.

\section{Materials and Methods}

\section{Test Media}

The following solutions were tested:

Cornea organ culture medium (CM I) (P04-09701; PAN-Biotech, Aidenbach, Germany): modified Eagle medium (MEM) with Earle salts, $2 \%$ fetal bovine serum, penicillin $\mathrm{G}(62.5 \mu \mathrm{g} / \mathrm{mL})$, streptomycin $(100 \mu \mathrm{g} / \mathrm{mL})$, and amphotericin $\mathrm{B}(2.5 \mu \mathrm{g} / \mathrm{mL})$

Cornea organ culture medium with dextran (CM II) (P0409702; PAN-Biotech): MEM) with Earle salts, 2\% fetal bovine serum, penicillin G $(62.5 \mu \mathrm{g} / \mathrm{mL})$, streptomycin $(100 \mu \mathrm{g} / \mathrm{mL})$, amphotericin B $(2.5 \mu \mathrm{g} / \mathrm{mL}$ ), and $6 \%$ dextran 500 (as it is used for detumescence of organ-cultured corneas)

Femoral head medium (FH): Ringer solution (2610813; Fresenius Kabi GmbH, Bad Homburg, Germany) containing $6.5 \mathrm{~g} \mathrm{NaCl}$, $0.42 \mathrm{~g} \mathrm{KCl}, 0.25 \mathrm{~g} \mathrm{CaCl}_{2} 2 \mathrm{H}_{2} \mathrm{O}$, and $1 \mathrm{~mol} \mathrm{NAHCO}_{3}$ dissolved in
$1 \mathrm{~L}$ of distilled water. The Ringer solution was used for thermal disinfection of femoral heads with the Marburg Bone Bank System Lobator SD-2 (Telos GmbH, Marburg, Germany) after standard processing in our tissue bank as described before [11], tested for sterility, and preserved at $-20^{\circ} \mathrm{C}$ until usage for testing.

\section{Culture Bottles}

BD BACTEC ${ }^{\mathrm{TM}}$ plastic blood culture bottles for clinical as well as industrial use and the automated test system BACTEC ${ }^{\mathrm{TM}} \mathrm{FX}$ were used (Becton Dickinson and Company, Sparks, NV, USA). For cornea organ culture medium with and without dextran we used BACTEC ${ }^{\mathrm{TM}}$ Plus Aerobic/F and Anaerobic/F bottles which contain nonionic adsorbing and cationic exchange resins that inactivate antibiotics [12] with the addition of $1 \mathrm{ml}$ of $\mathrm{BBL}^{\mathrm{TM}}$ penicillinase concentrate (211899; Becton Dickinson). One milliliter of enzyme is sufficient for inactivation of $1 \times 10^{7} \mathrm{IU}$ penicillin G. Our own investigations [unpubl. data] showed insufficient reduction of penicillin by the resins alone. For FH the BD BACTEC ${ }^{\text {TM }}$ Standard Aerobic/F and Anaerobic/F bottles were inoculated.

\section{Test Strains}

The following microbial strains were used as recommended for validation by the Ph. Eur.: Staphylococcus aureus, Pseudomonas aeruginosa, Bacillus subtilis, Candida albicans, Aspergillus brasiliensis, and Clostridium sporogenes. Cutibacterium acnes (formerly Propionibacterium acnes), S. epidermidis and Enterococcus faecalis (Table 1) were chosen additionally because of their relevance for the tissue processed in our multitissue bank. All strains were obtained from Becton Dickinson.

\section{Inoculum Preparation}

Lyophilized pellets were rehydrated on Columbia agar plates with sheep blood plus (CBA, PB5039A; Oxoid, Wesel, Germany) and Sabouraud dextrose agar plates (254039; Becton Dickinson) and grown under optimal growth conditions according to the manufacturer's instructions (Table 1). The cultures were visually controlled for pure growth and the typical morphology of the colonies and resuspended in isotonic saline solution. Concentrations were adjusted to a turbidity of 1.0 according to the McFarland turbidity standard using a DEN-1 McFarland densitometer (Biosan, Riga, Latvia), which equates to a CFU of $3 \times 10^{6} / \mathrm{mL}$ for C. albicans and 
Table 2. Preparation of test bottles.

\begin{tabular}{|c|c|c|c|c|c|}
\hline Test bottle & BD BACTEC ${ }^{\mathrm{TM}}$ bottle & Medium & $\begin{array}{l}\text { Volume, } \\
\mathrm{mL}\end{array}$ & $\begin{array}{l}\text { Enzyme }^{1} \text {, } \\
\mathrm{mL}\end{array}$ & $\begin{array}{l}\text { Test strain, } \\
\mu \mathrm{L}\end{array}$ \\
\hline $\mathrm{CM} \mathrm{I/II} \mathrm{aerobic}$ & Plus Aerobic/F & CM I/CM II & $8.5 \pm 0.5$ & 1 & 100 \\
\hline $\mathrm{CM} \mathrm{I} / \mathrm{II}$ anaerobic & Plus Anaerobic/F & CM I/CM II & $8.5 \pm 0.5$ & 1 & 100 \\
\hline FH aerobic & Standard Aerobic/F & $\mathrm{FH}$ & $9.5 \pm 0.5$ & - & 100 \\
\hline FH anaerobic & Standard Anaerobic/F & $\mathrm{FH}$ & $6.5 \pm 0.5$ & - & 100 \\
\hline \multicolumn{6}{|l|}{ Negative controls } \\
\hline (-) control aerobic CM I/II & Plus Aerobic/F & $\begin{array}{l}\text { CM I/CM II/ } \\
\text { saline solution }\end{array}$ & $8.5 \pm 0.5$ & 1 & - \\
\hline (-) control anaerobic CM I/II & Plus Anaerobic/F & $\begin{array}{l}\mathrm{CM} \text { I/CM II/ } \\
\text { saline solution }\end{array}$ & $8.5 \pm 0.5$ & 1 & - \\
\hline (-) control aerobic FH & Standard Aerobic/F & $\mathrm{FH} /$ saline solution & $9.5 \pm 0.5$ & - & - \\
\hline$(-)$ control anaerobic FH & Standard Anaerobic/F & $\mathrm{FH} /$ saline solution & $6.5 \pm 0.5$ & - & - \\
\hline \multicolumn{6}{|l|}{ Positive controls } \\
\hline (+) control aerobic CM I/II & Plus Aerobic/F & saline solution & $8.5 \pm 0.5$ & 1 & 100 \\
\hline (+) control anaerobic CM I/II & Plus Anaerobic/F & saline solution & $8.5 \pm 0.5$ & 1 & 100 \\
\hline (+) control aerobic FH & Standard Aerobic/F & saline solution & $9.5 \pm 0.5$ & - & 100 \\
\hline (+) control anaerobic FH & Standard Anaerobic/F & saline solution & $6.5 \pm 0.5$ & - & 100 \\
\hline
\end{tabular}

The test bottles are named after their injected medium or as controls for the corresponding medium. Culture media I and II contain antibiotics and are injected in aerobic and anaerobic BD BACTEC ${ }^{\mathrm{TM}}$ Plus bottles and penicillinase is added. The femoral head medium is injected in aerobic and anaerobic standard bottles without resins. The manufacturer's recommendations for the addition of test media are: 8-10 mL for Plus Aerobic/F, Plus Anaerobic/F, and Standard Aerobic/F bottles and 5-7 mL for Standard Anaerobic/F bottles. $(-)$ negative; (+) positive. ${ }^{1}$ Penicillinase $1 \mathrm{~mL}$ inactivates $1 \times 10^{7} \mathrm{IU}$ penicillin G.

$3 \times 10^{7} / \mathrm{mL}$ for the other strains, except A. brasiliensis. Dilutions were prepared with saline solution to $300 \mathrm{CFU} / \mathrm{mL}$. One hundred microliters of this concentration were added to the blood culture bottles to obtain a spiking of 10-100 microorganisms. For A. brasiliensis, which cannot be prepared according to McFarland, an area of $2 \mathrm{~cm}^{2}$ of fungal growth presenting a fruiting body and mycelium was taken from the agar plate, resuspended in $10 \mathrm{~mL}$ of saline solution, and diluted by a factor of $10^{-4}$. The CFU was verified by plating $100 \mu \mathrm{L}$ of each strain on agar plates (Table 1). The used test media were monitored for sterility at the end of each preparation.

\section{Preparation of Test Bottles}

At first $6.5-9.5 \mathrm{~mL}$ of the test medium were injected into the blood culture bottles, depending on the manufacturer's recommendations for the optimal volume in the different bottle types (Table 2). For BACTEC ${ }^{\text {TM }}$ Plus Aerobic/F and Anaerobic/F bottles $1 \mathrm{~mL}$ of the ready-to-use penicillinase was added additionally and the bottles were incubated for $1 \mathrm{~h}$ at room temperature (RT). For negative controls only the respective test medium with the addition of penicillinase was transferred into the bottles; for positive controls instead of test media saline solution $(0.9 \% \mathrm{NaCl}$ Ecotainer at 1,000 mL; B. Braun Melsungen AG, Melsungen, Germany), and for "Plus bottles" also penicillinase, was added. All bottles except for the negative controls were spiked with $100 \mu \mathrm{L}$ of the diluted test strain. All preparations were done in duplicate and under aseptic conditions in a grade A laminar airflow cabinet (Hera Safe HS 15; Kendro Laboratory Products GmbH, Hanau, Germany) under controlled conditions at $21.0 \pm 1.5^{\circ} \mathrm{C}$ with no other activity conducted at the same time. Due to ongoing delivery problems with penicillinase, one duplicate of the strains S. aureus, A. brasiliensis, and C. sporogenes was incubated with penicillinase; the other bottle was incubated without penicillinase. As expected, this had an immense impact on the results for $S$. aureus and no effect at all for A. brasiliensis and C. sporogenes. However, to follow the study protocol and good scientific practice, none of the results of the duplicates (of these strains) was included in the data for this paper.

Following the inoculation, 2 groups of test bottles were placed into the BACTEC ${ }^{\mathrm{TM}} \mathrm{FX}$ unit at $30.0 \pm 1.5^{\circ} \mathrm{C}$ and $35 \pm 1.5^{\circ} \mathrm{C}$ and a third group was left at RT $\left(21.0 \pm 1.5^{\circ} \mathrm{C}\right)$ with constant agitation for incubation. The BACTEC ${ }^{\mathrm{TM}} \mathrm{FX}$ unit uses a $\mathrm{CO}_{2} /$ fluorescin method for constant automated reading. The bottles were left in the BACTEC ${ }^{\mathrm{TM}} \mathrm{FX}$ unit until detection of microbiological growth was recorded automatically or for a maximum of 16 days. The time point of microbiological growth was recorded automatically and later evaluated and calculated in minutes after insertion into the BACTEC $^{\text {TM }}$ FX unit. The bottles incubated at RT were macroscopically controlled twice per working day, and once a day on the weekend, for a maximum of 16 days. When microbiological growth was visually detected, the timespan was recorded and later evaluated in hours after first start of agitation.

Through subculturing on CBA or Sabouraud dextrose agar plates the pure culture and typical morphology of the colonies were proven for all positive blood culture bottles and the identity of the microorganism was verified by an independent laboratory (Labor Berlin, Berlin, Germany). All negative test bottles were examined in the same way to prove the absence of microorganisms (plating of $100 \mu \mathrm{L}$ per bottle and incubation of the agar plates for 2-5 days before evaluation, depending on the test strain).

\section{Results}

All of the results are shown in Figure 1-3, indicating the time to detection (TTD) of microbial growth in hours, either identified by the automated BACTEC ${ }^{\mathrm{TM}}$ FX unit 


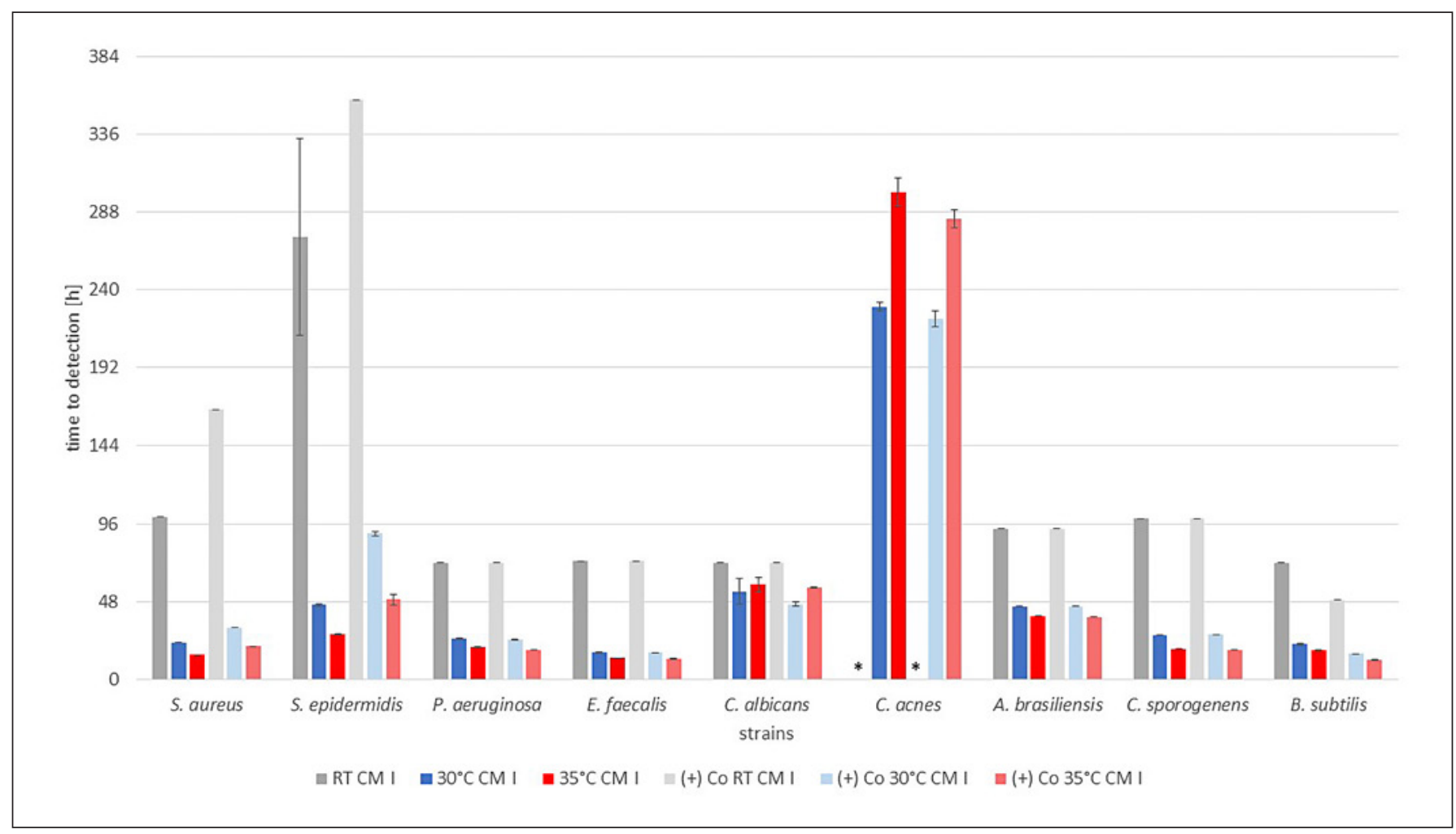

Fig. 1. TTD of the test strains with organ cornea culture medium without dextran (CM I) in hours. Results at RT and 30 and $35^{\circ} \mathrm{C}$ with the corresponding positive control, i.e., (+) Co, afterwards are shown for each strain. TTD is given as an average of the duplicates \pm SD. ${ }^{*}$ No growth detected after 16 days $(384 \mathrm{~h})$.

( 30 and $35^{\circ} \mathrm{C}$ ) or individually by a person for RT. In each graph results are shown for all strains and a single test medium, with bars indicating the TTD at RT (grey), $30^{\circ} \mathrm{C}$ (blue), and $35^{\circ} \mathrm{C}$ (red), followed by the corresponding positive controls in the same order.

All positive controls showed confirmed pure culture growth with saline solution instead of the test media at 30 and $35^{\circ} \mathrm{C}$ with the BACTEC ${ }^{\mathrm{TM}} \mathrm{FX}$ unit. At RT for all media the positive controls for $C$. acnes showed no growth within 16 days. Overall, the TTD for the positive controls was similar to all test media for every microorganism (shown in Fig. 1-3). All negative controls remained sterile for 16 days and showed no growth on agar plates thereafter (data not shown).

In the presence of CM I, all test strains were detected at all temperatures, with the exception of $C$. acnes at RT. The TTD was shortest at $35^{\circ} \mathrm{C}$, delayed at $30^{\circ} \mathrm{C}$ and even later at RT for 7 out of the 9 strains. C. albicans was detected at comparable time points at 35 and $30^{\circ} \mathrm{C}$ and only a little later at RT. C. acnes was detected first at $30^{\circ} \mathrm{C}$ and later at $35^{\circ} \mathrm{C}$. For the positive controls the chronology for the detection of microbial growth was similar and also the TTD was comparable (shown in Fig. 1).

For incubation with CM II the results are almost identical to the results with CM I. The same 7 test strains grew fastest at $35^{\circ} \mathrm{C}$ and more slowly at $30^{\circ} \mathrm{C}$, and detection was delayed at RT. C. acnes and C. albicans had their shortest TTD at $30^{\circ} \mathrm{C}$ and grew more slowly at $35^{\circ} \mathrm{C}$. C. albicans was detected at similar time points at $35^{\circ} \mathrm{C}$ and RT. No growth was detected for C. acnes at RT. The corresponding positive controls showed similar time patterns (shown in Fig. 2).

For the FH all test strains except for C. acnes were detected first at $35^{\circ} \mathrm{C}$, later at $30^{\circ} \mathrm{C}$, and last at RT. The positive controls showed comparable TDD. C. acnes was only detectable if incubated at $35^{\circ} \mathrm{C}$. There was no growth at $30^{\circ} \mathrm{C}$ with $\mathrm{FH}$, although $\mathrm{C}$. acnes was detectable in the positive control at $30^{\circ} \mathrm{C}$ (shown in Fig. 3).

With all 3 media $S$. epidermidis showed a more than 5 times higher TTD at RT compared to 30 and $35^{\circ} \mathrm{C}$. The averages $( \pm \mathrm{SD})$ of all registered TTD for all 3 test media combined are $106 \mathrm{~h}( \pm 69.77)$ for RT, $45.17 \mathrm{~h}( \pm 49.39)$ for $30^{\circ} \mathrm{C}$, and $50.02 \mathrm{~h}( \pm 72.56)$ for $35^{\circ} \mathrm{C}$. When no growth was detected the test strain was not included in the calculation.

\section{Discussion/Conclusion}

In our study we compared different temperatures for microbiological testing and 3 different media, which undergo testing on a frequent basis in our multitissue bank. CM I and CM II are used for cultivation of postmortem 


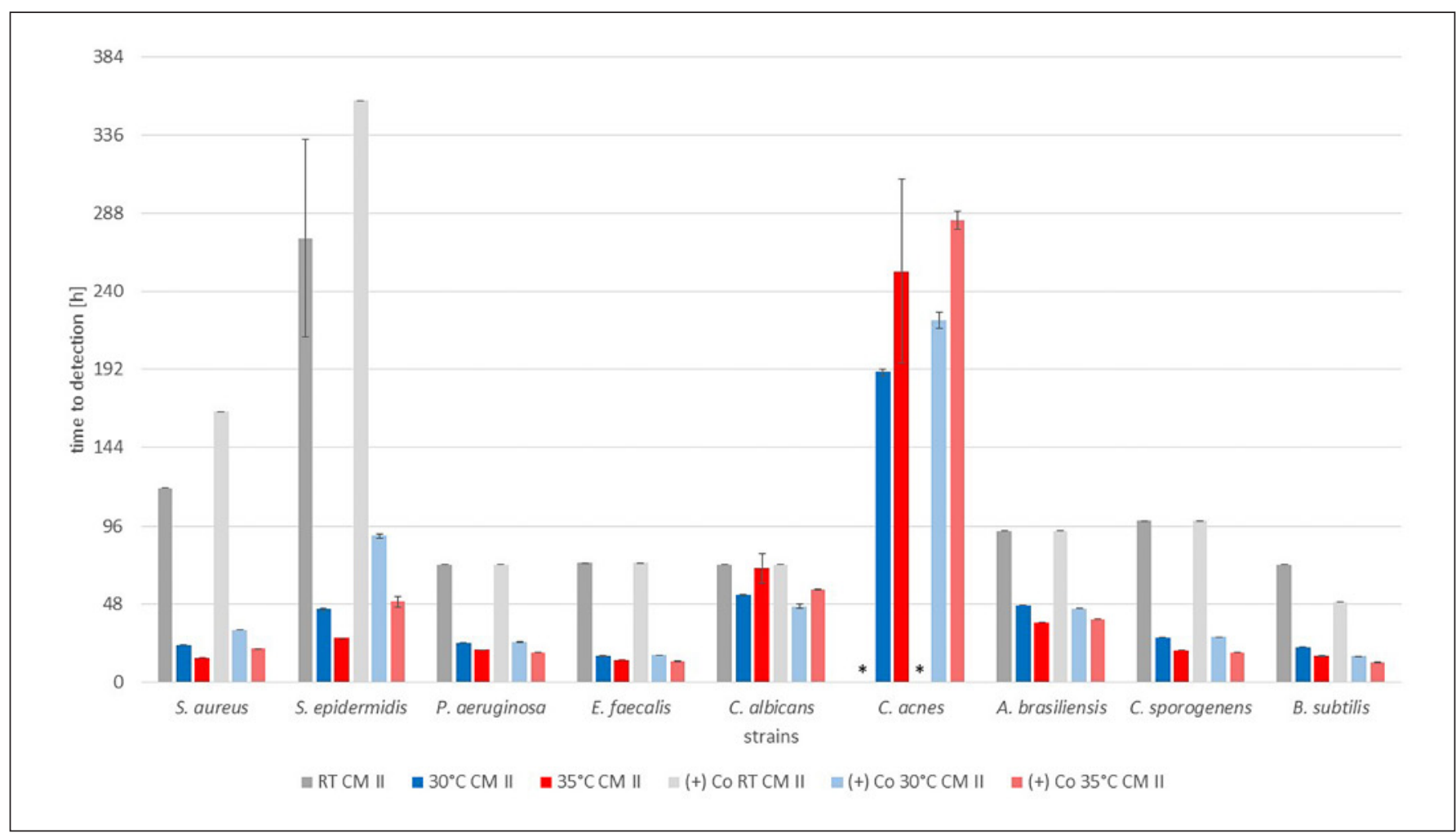

Fig. 2. TTD of the test strains with organ cornea culture medium with dextran (CM II) in hours. Results at RT and 30 and $35^{\circ} \mathrm{C}$ with the corresponding positive control, i.e., (+) Co, afterwards are shown for each strain. TTD is given as an average of the duplicates \pm SD. ${ }^{*}$ No growth detected after 16 days $(384 \mathrm{~h})$.

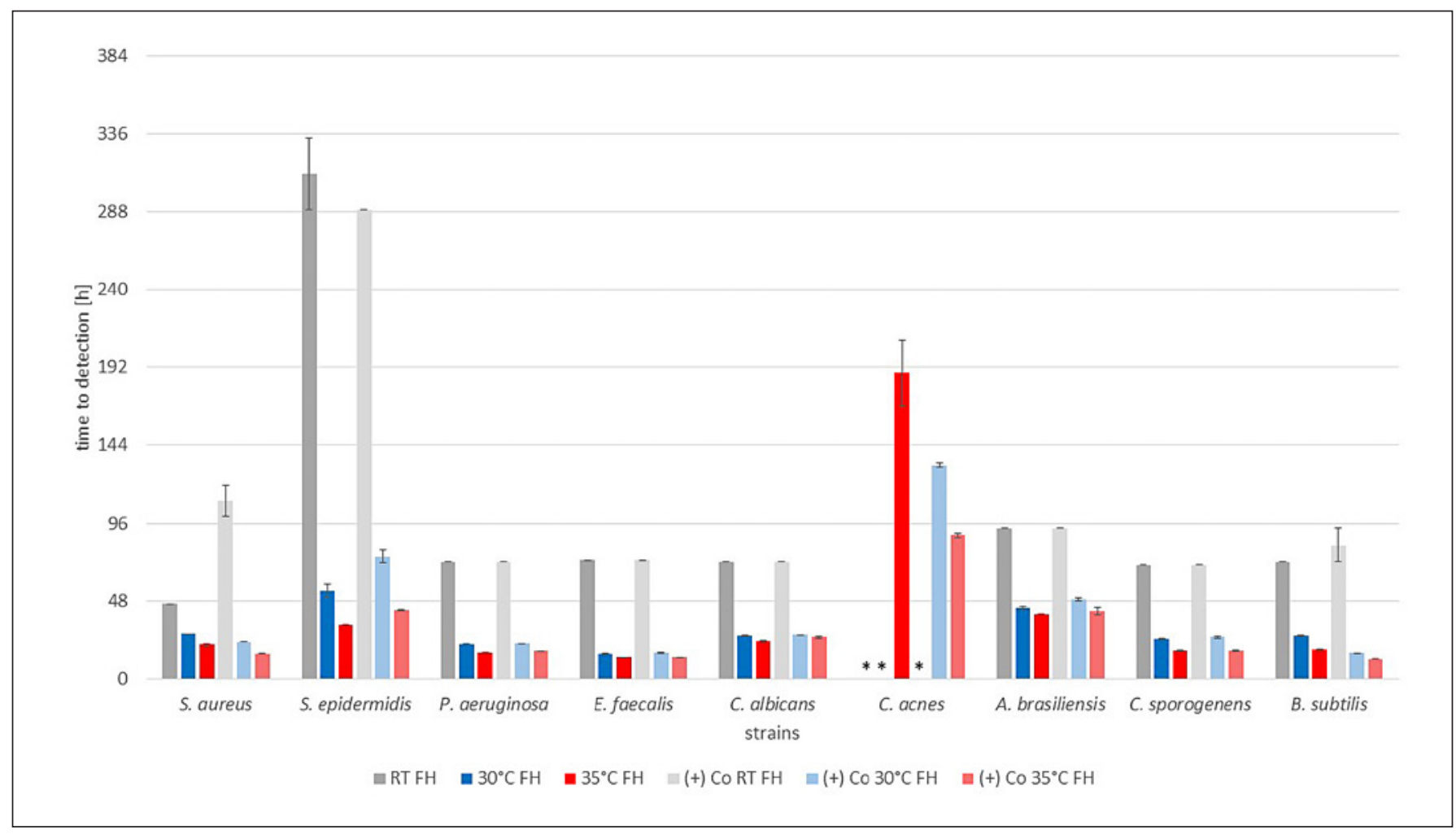

Fig. 3. TTD of the test strains with $\mathrm{FH}$ in hours. Results at $\mathrm{RT}$ and 30 and $35^{\circ} \mathrm{C}$ with the corresponding positive control, i.e., (+) Co, afterwards are shown for each strain. TTD is given as an average of the duplicates $( \pm$ SD). * No growth detected after 16 days $(384 \mathrm{~h})$. 
donated corneas. Studies have shown that donor corneas are significantly contaminated with microorganisms due to multiple reasons even after disinfection of the whole globe [13]. Therefore, antibiotic treatment is a helpful method to reduce the risk for the recipient [14]. But with antibiotic treatment the risk must be considered that some microorganisms may survive decontamination but are not detected by postdecontamination microbiological testing, thus creating a false-negative result. This creates further challenges of complete antibiotic inactivation while the microbiological testing is done.

Femoral head solution is created in a terminal sterilization process on tissue harvested from living donors. Thus, the initial contamination rate is very low [15]. The donated femoral head is put into Ringer solution and during an interval of $94 \mathrm{~min}$ a heat decontamination process takes place. After this process many substances which are physiologically inside the femoral head will be dissolved in Ringer solution. When the femoral head solution is microbiologically tested it already has a strong turbidity, which hampers the performance of multiple microbiological test systems [10].

Blood culture bottles are an established microbiological test system with the advantage of automated growth detection and the option to use culture bottles containing resins for inactivation of antibiotics [16]. Therefore, a wide range of fluids can be tested. Sterility controls of cornea organ culture media have been evaluated and validated in the past, and studies recommend the addition of penicillinase for sufficient inactivation of antibiotics [8].

In our study for each test medium all microorganisms were detected at a single temperature, which underlines the suitability and safety of the test system for microbiological control. In cornea organ culture medium without dextran (CM I), growth of all strains could be detected within 10 days at $30^{\circ} \mathrm{C}$ and within 13 days at $35^{\circ} \mathrm{C}$, with $C$. acnes being the main reason for the difference. The other 8 strains were detected within 3 days at both temperatures. The positive controls showed almost exactly the same results as the bottles spiked with CM I. Obviously, the culture medium has no negative influence on the growth of microorganisms and deactivation of the antibiotics was successful.

Cornea organ culture medium with dextran (CM II) has the same properties as CM I with the addition of $6 \%$ dextran. The detection of all microorganisms was successful within 8 days at $30^{\circ} \mathrm{C}$ and within 13 days at $35^{\circ} \mathrm{C}$, again with $C$. acnes being the reason for the difference. All other strains were detected within 3 days, except for a single blood culture bottle spiked with C. albicans at $35^{\circ} \mathrm{C}$ with a TTD of $78.5 \mathrm{~h}$. All of the results for CM II are very similar to those for CM I. Dextran does not seem to have an influence on bacterial growth or interact with antibiotics or penicillinase in a relevant dimension.
In the case of $\mathrm{FH}$, we expected that the initial turbidity might have an influence on the outcome. To detect microbial growth at the earliest possible point of time, the clarity of the medium is an essential factor. Some microorganisms can be macroscopically detected while others increase the turbidity of the medium. But with an already high turbidity the detection of microbial growth is essentially more complicated in any case. Microbial growth could be detected for all strains at $35^{\circ} \mathrm{C}$ within 9 days. At $\mathrm{RT}$ and $30^{\circ} \mathrm{C}$ no growth of $\mathrm{C}$. acnes was detected within 16 days. The TTD for each strain and each temperature was similar to the values for the positive controls executed with saline solution. The BACTEC ${ }^{\mathrm{TM}} \mathrm{FX}$ unit was not compromised by the initial turbidity, as it has no influence on the $\mathrm{CO}_{2}$ /fluorescein method.

The results for all 3 test solutions are similar. C. acnes is known to grow slowly and was for each medium the strain that was most difficult to detect. In general, the selected strains showed a longer TTD at RT and faster as well as more thorough, regarding $C$. acnes, detection at the warmer temperatures 30 and $35^{\circ} \mathrm{C}$.

In 2015 Thomasen et al. [8] evaluated a new protocol for sterility controls of corneal culture medium and demonstrated detection times for P. aeruginosa, B. subtilis, $S$. aureus, C. sporogenes, C. acnes, and C. albicans in CM I using the BacT/Alert system. Thomasen et al. [8] used $22^{\circ} \mathrm{C}$ for aerobic microorganisms and $32^{\circ} \mathrm{C}$ for anaerobic, mesophilic microorganisms. Noticeable are the TTD for aerobic organisms. They are mostly similar to the TTD at RT in our study, which were all surpassed with shorter TTD at 30 and $35^{\circ} \mathrm{C}$. The growth detection for fungi by Thomasen et al. [8] is again comparable to our results at RT. For C. albicans TTD at 30 and $35^{\circ} \mathrm{C}$ are in the same range; for $A$. brasiliensis the TTD at the warm temperatures were only half as long.

In 2012 Schroeter at al. [7] validated the BACTEC ${ }^{\text {TM }}$ FX unit for tissue preparations testing the 6 strains recommended by the Ph. Eur. The inoculated blood culture bottles were incubated for $12 \mathrm{~h}$ at $20 \pm 4^{\circ} \mathrm{C}$ and afterwards transferred into the BACTEC ${ }^{\mathrm{TM}} \mathrm{FX}$ unit at $35 \pm 1{ }^{\circ} \mathrm{C}$. The results are earlier TTD for FH (Ringer), CM I, and CM II. The initial incubation of $12 \mathrm{~h}$ was not added to the presented TTD in the results of this study. Considering this information, the TTD for all media are comparable to the results we present. Noticeable in that study was that, while working with resin-containing blood culture bottles but without penicillinase, S. aureus showed no growth in the presence CM I and CM II. This underlines the need for additional penicillinase when working with cornea medium containing antibiotics.

There certainly are many microorganisms that have their optimal growth, and thus optimal detection, at lower temperatures, but the selected strains included all strains recommended by the $\mathrm{Ph}$. Eur. They already show 
a broad bandwidth of microbial diversity with aerobic and anaerobic bacteria as well as fungi, and they are most relevant regarding a possible contamination of human tissue. Furthermore, bacteria were included in this study because of their relevance for eye infections and postoperative infections and their overall relevance for contamination in hospitals. Other bacteria that might be present on the tissue of interest and are not being detected while performing microbiological testing are probably not of high clinical relevance after transplantation of the tissue $[17,18]$. Otherwise past and current studies would have identified these microorganisms, as occurred, e.g., with the herpes virus for corneal tissue transplants [19-21]; posttransplantation infections would be of a much higher relevance.

Also tissue donors are screened for infections of the tissue that might be donated, and a possible infection is always a contraindication for tissue donation. The microbial spectrum that is most commonly seen in eye infections, conjunctivitis, or osteomyelitis differs from the microbial spectrum of this study. It might be interesting to test other strains for the optimal growth temperatures for clinical purposes, especially as infections of the eye arise at lower temperatures than body temperature.

Testing at a single temperature might have more advantages. The tests carried out at 30 and $35^{\circ} \mathrm{C}$ were performed by the semiautomated BACTEC ${ }^{\mathrm{TM}} \mathrm{FX}$ unit. The automated detection with constant surveillance of the blood culture bottles guarantees objectivity on the decision for positive growth at the earliest possible time and the "human factor" becomes minimized as a possible threat for the tissue's safety. In Germany, the majority of the more than 20 tissue banks working with thermal disinfected femoral heads and more than 20 tissue banks culturing corneas belong to hospitals. To provide the patient's diagnostic routine in these hospitals, the proper laboratories are already equipped with automated systems working at temperatures between 30 and $37^{\circ} \mathrm{C}$ so that the tissue banks can profit by these systems.

In the presented validation study $35^{\circ} \mathrm{C}$ was shown to be the temperature with the fastest growth of the majority of microorganisms within 14 days. From our data, we conclude that the requirements for a successful method suitability test are fulfilled for the semiautomated blood culture bottle system with the BACTEC ${ }^{\mathrm{TM}} \mathrm{FX}$ unit for the media and test strains used. It must be kept in mind that the method for microbiological testing in a tissue bank should be best suited not only for the detection of Ph. Eur. test strains but also for the detection of other relevant microbial contaminants. Therefore, one incubation condition may not be best suited for microbiological control testing of different tissues, as the spectra of contaminants may vary. In general, test conditions should be as broad as possible to suite the worst-case scenarios, which may change over time and must be constantly reassessed. For instance, fungal contaminants of the cornea, which are important in endophtalmitis or keratitis, frequently display growth at $25^{\circ} \mathrm{C}$ but grow slowly or cease to grow at elevated temperatures. More studies are needed to further optimize the microbiological testing of tissue preparations regarding improved patient safety.

\section{Statement of Ethics}

Does not apply.

\section{Conflict of Interest Statement}

The authors have no conflicts of interest to declare.

\section{Funding Sources}

This research was not funded by exterior sources.

\section{Author Contributions}

Study theme: Axel Pruß. Study design: Eva Schulz and Frithjof Herrlinger. Experiments: Frithjof Herrlinger, Eva Schulz, and Tino Schulz. Data analysis: Frithjof Herrlinger, Eva Schulz, and Tino Schulz. Writing of this paper: Frithjof Herrlinger. Revision of this paper: Eva Schulz and Axel Pruss.

\section{References}

1 Schroeter J, Maier P, Bednarz J, Bluthner K, Quenzel M, Pruss A, et al. Procedural guidelines. Good tissue practice for cornea banks. Ophthalmologe. 2009;106(3):265-74, 76.

2 Pruss A, Göbel UB, Pauli G. Infections associated with musculoskeletal-tissue allografts. $\mathrm{N}$ Engl J Med. 2004 Sep;351(13):1358-60.

3 Kainer MA, Linden JV, Whaley DN, Holmes HT, Jarvis WR, Jernigan DB, et al. Clostridium infections associated with musculoskeletal-tissue allografts. N Engl J Med. 2004 Jun; 350(25):2564-71
4 Kuehnert MJ, Clark E, Lockhart SR, Soll DR, Chia J, Jarvis WR. Candida albicans endocarditis associated with a contaminated aortic valve allograft: implications for regulation of allograft processing. Clin Infect Dis. 1998 Oct; 27(4):688-91.

5 Wilson ML. Blood cultures. Introduction. Clin Lab Med. 1994 Mar;14(1):1-7.

6 Wilson ML, Weinstein MP. General principles in the laboratory detection of bacteremia and fungemia. Clin Lab Med. 1994 Mar;14(1): 69-82.
7 Schroeter J, Wilkemeyer I, Schiller RA, Pruss A. Validation of the Microbiological Testing of Tissue Preparations Using the BACTEC ${ }^{\mathrm{TM}}$ Blood Culture System. Transfus Med Hemother. 2012 Dec;39(6):387-90.

8 Thomasen H, Mosel F, Steuhl KP, Meller D. Evaluation of a new protocol for sterility controls of corneal culture medium. Cell Tissue Bank. 2015 Sep;16(3):343-50.

9 European Directorate for the Quality of Medicines. European Pharmacopoeia. Strasbourg; EDQM: 2019. 
10 European Committee (Partial Agreement) on Organ Transplantation (CD-P-TO). Guide to the quality and safety of tissues and cells for human application. 4th ed. Strasbourg: EDQM; 2019. p. 143-55

11 Pruss A, Seibold M, Benedix F, Frommelt L, von Garrel T, Gürtler L, et al. Validation of the 'Marburg bone bank system' for thermodisinfection of allogenic femoral head transplants using selected bacteria, fungi, and spores. Biologicals. 2003 Dec;31(4):28794.

12 Appelbaum PC, Beckwith DG, Dipersio JR Dyke JW, Salventi JF, Stone LL. Enhanced detection of bacteremia with a new BACTEC resin blood culture medium. J Clin Microbiol. 1983 Jan;17(1):48-51.
13 Röck D, Wude J, Bartz-Schmidt KU, Yoeruek E, Thaler S, Röck T. Factors influencing the contamination rate of human organ-cultured corneas. Acta Ophthalmol. 2017 Dec; 95(8):e706-12.

14 Pels L. Organ culture: the method of choice for preservation of human donor corneas. $\mathrm{Br}$ J Ophthalmol. 1997 Jul; 81(7): 523-525.

15 Varettas K, Taylor P. Bioburden assessment of banked bone used for allografts. Cell Tissue Bank. 2011 Feb;12(1):37-43.

16 Skenderi Z, Giurgola L, Gatto C, D'Amato Tóthová J, Pruß A, Schroeter J. Increased sensitivity of microbiological testing of cornea organ culture medium by additional resin treatment. BMJ Open Ophthalmol. 2018 Nov; 3(1):e000173.
17 Albon J, Armstrong M, Tullo AB. Bacterial contamination of human organ-cultured corneas. Cornea. 2001 Apr;20(3):260-3.

18 Farrell PL, Fan JT, Smith RE, Trousdale MD. Donor cornea bacterial contamination. Cornea. 1991 Sep;10(5):381-6.

19 Cleator GM, Klapper PE, Dennett C, Sullivan AL, Bonshek RE, Marcyniuk B, et al. Corneal donor infection by herpes simplex virus: herpes simplex virus DNA in donor corneas. Cornea. 1994 Jul;13(4):294-304.

20 Cockerham GC, Krafft AE, McLean IW. Herpes simplex virus in primary graft failure. Arch Opthalmol. 1997 May;115(5):586-9.

21 Remeijer L, Maertzdorf J, Doornenbal P, Verjans GM, Osterhaus AD. Herpes simplex virus 1 transmission through corneal transplantation. Lancet. 2001 Feb;357(9254):442. 